\title{
NUMERICAL STUDY OF THE IMPACT ON COOLING BEHAVIOUR OF VENT-HOLES DESIGN OF FRUIT PACKAGING BOXES
}

\author{
JOÃO CURTO', ADHIYAMAN ILANGOVAN ${ }^{1}$, PEDRO D. GASPAR ${ }^{1,2}$, PEDRO D. \\ SILVA $^{1,2}$, NANCI ALVES ${ }^{3}$ \\ ${ }^{1}$ University of Beira Interior \\ Rua Marquês de D'Ávila e Bolama, Covilhã, 6201-001, Portugal, \\ curto.joao5@gmail.com, adhi.ilangovan@ubi.pt,dinis@ubi.pt, dinho@ubi.pt,www.ubi.pt \\ ${ }^{2}$ C-MAST - Center for Mechanical and Aerospace Science and Technologies \\ Rua Marquês de D’Ávila e Bolama, Covilhã, 6201-001, Portugal \\ dinis@ubi.pt, dinho@ubi.pt, http://www.aerospace.ubi.pt/ \\ ${ }^{3}$ TJ Moldes \\ Embra - P.O. BOX 198, Marinha Grande, 2431-966, Portugal \\ nalves@tj-moldes.pt,
}

Key words: Packaging boxes design, Airflow and thermal performance, Shelf-life extension, Experimental tests, Parametric study, Computational Fluid Dynamics.

\begin{abstract}
Fruit packaging is highly impactful in temperature and humidity distribution for post-harvest fresh fruit cooling and conservation. It is also the most flexible part on fruit production and should be able to increase shelf-life by deterring adverse conditions. However, packaging thermal performance during cold storage where it is displayed to the internal airflow is influenced by the size and position of vent-holes. In this study airflow inside the fruit box and fruits thermal behaviour are evaluated in order to determine differences between three prototype package models. Models differ from each other by the holes' configuration and size. Furthermore, models' performance is compared to a commercially available box of similar dimensions. Experimental tests were carried out in three stacked boxes, equipped with temperature and humidity sensors, and placed inside a refrigeration chamber with forced air cooling. Packaging box CFD models of each prototype wall were developed to predict the airflow and heat transfer during storage in a refrigeration chamber. Models predictions were validated by experiments results. According with simulation results, all prototypes have better thermal performance than the commercial model. However, significant performance differences between prototype walls were found. Fewer, larger and strategically distributed vent-holes allow a better result in terms of temperature homogeneity inside the box and significant reduction in fruit cooling times.
\end{abstract}

\section{INTRODUCTION}

Temperature is the most important factor affecting post-harvest fruit quality. To stave off the decrease of quality, fresh produce is subjected to precooling, most often to a stream of cool air that rapidly decreases the temperature to a point near the desired conservation 
condition, removing field heat and safeguarding the quality decay of commodities [1-7]. The maintenance of adequate temperature slows down the biochemical processes responsible for senescence [8]. Fruit remains physiologically active after harvest resulting in the reduction of quality $[9,10]$. Adequate cooling is of critical importance when dealing with post-harvest perishable fruits as the mentioned physiological phenomena generates heat that can lead to premature and excessive ripening [11] and the development of colony forming units of microorganisms. Thus, it becomes clear that proper temperature management is necessary to ensure perishable food quality and safety [12]. As forced air cooling is the most common process used to cool post-harvest fruit, it also becomes clear the importance of this stage in the food chain and its necessity to be as effective and uniform as possible [13, 14].

Cooling heterogeneity can lead to damage in the produce due to some locations becoming colder or hotter than ideal $[1,11]$ and it is difficult to evaluate using average values [9]. One of the factors affecting cooling uniformity during precooling is the presence of turbulent airflow due to the shape of food products and the packaging $[15,16]$. Therefore, packaging should be designed to prevent heterogeneity by adjusting vent size and position to the necessities of the food products $[6,17]$. However, perfect cooling uniformity is impossible to operational and environmental conditions as well as biological variance of perishable food products $[9,10]$. Virtual cold chain simulation clearly demonstrates the negative effects of cooling heterogeneity in the quality of food products [14]. Heterogeneity can also be caused by the presence of preferential pathways for airflow, in which the food products aligned with

\section{the path can be properly cooled, but the misaligned ones cannot [18, 19}

Out of all the factors significant as it direct products, thus directly studied the impact of th that infuence precod
mpacting cooling time
design of the vents in
that designs includi
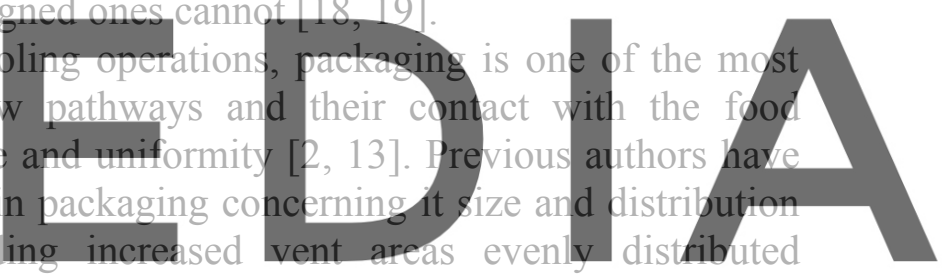

throughout the packaging perform better and with more uniformity than those who do not. by

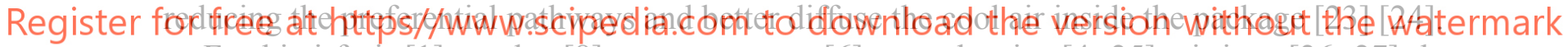

For kiwi fruit [1], apples [8], pomegranates [6], strawberries [4, 25], citrines [26, 27] the significant impact of package geometry on cooling and throughput [28] has been demonstrated.

Fortunately, packaging is one of the easiest parameters to change in post-harvest cold chain as it is not particularly expensive or overburdened with regulations [7]. Also, significantly less expensive is the use of CFD simulations per opposition to experimental tests and computational capabilities becomes less expensive, simulation become more attractive as it can provide accurate spatial and temporal predictions with a resolution and consistency that would be extremely difficult to achieve through experimentation $[2,6,7,9,10,26,28,29]$.

In this study, three types of fruit packaging boxes are studied concerning cooling efficiency and uniformity. The boxes vary only in the size, position, and shape of the vent holes. This numerical study continues the analysis started in previous works [30-34] where the evaluation of different fruit packaging box designs was developed, leading to a set of best performance packaging boxes. This study presents the multi-parameter approach using transient CFD models of the three best different packaging boxes with different vent shape, size, and location to predicts the airflow and temperature distribution during the cooling phase. 


\section{EXPERIMENTAL SETUP}

A stack of three boxes was placed inside a cooling chamber. Each box measures 600x400x90 mm and contains 35 product simulators, thus totaling one 105 fruit simulators. All simulators are spherical and have a diameter of $70 \mathrm{~mm}$ and are placed in a staggered pattern inside the alveoli being distanced from one another by $4 \mathrm{~mm}$. Five of the simulators are made of agar-gel and the remainder is made of polystyrene. One agar-gel simulator was placed in the center of each box and, in the middle box, the remaining two simulators were placed near the corners, one in near the bottom right corner and the other near the top left corner.

The models of box walls were named A, B and C (see Figure 1). Model A consists of a wall with cone like shaped holes for air circulation. Model B consists of a wall with four large openings located near the top of the box. Model C consists of two walls, an anterior wall with 36 small openings and a posterior wall with the same number of larger openings. The experimental tests are described in detail in [34]

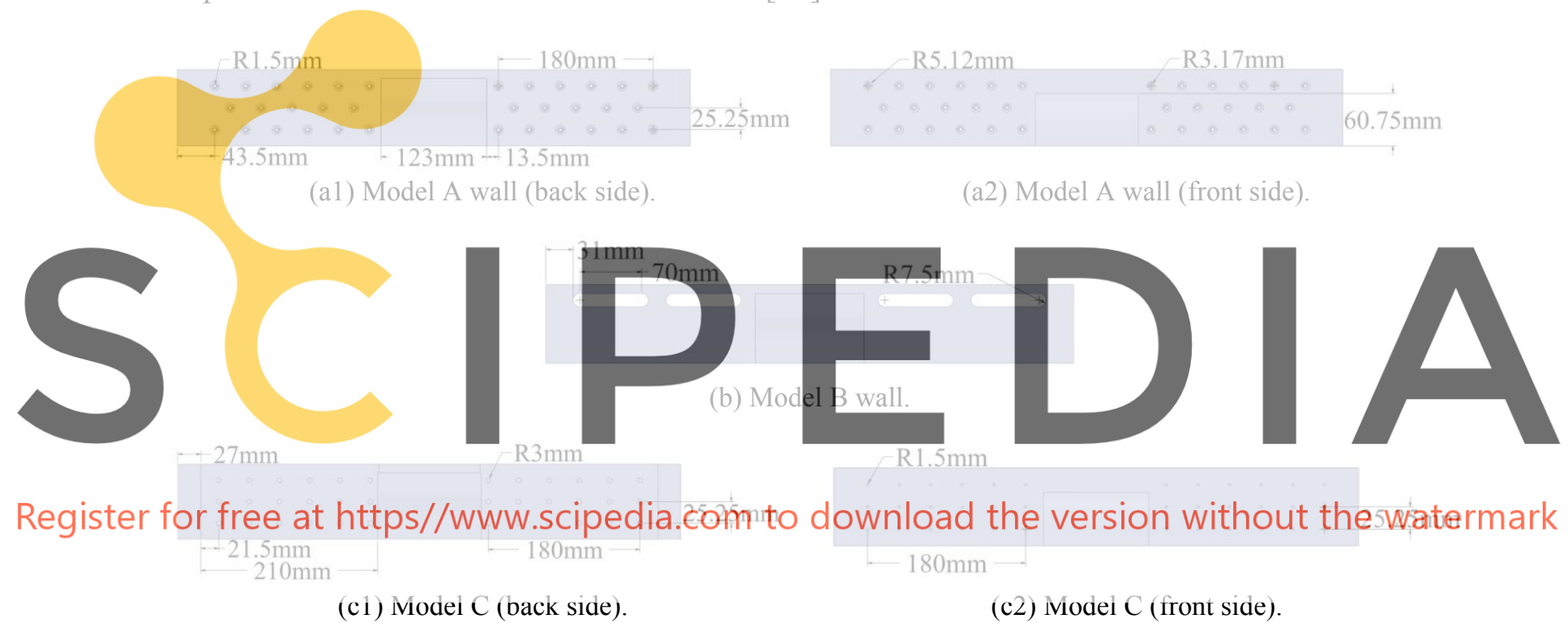

Figure 1: Box walls configuration of models.

The chamber was left open overnight to warm up to ambient temperature, which determined the starting point for cooling. After all equipment reaches thermal equilibrium with the environment, the chamber is closed, and the cooling operation starts. The stack of boxes was placed near the center of the chamber and cold air was moved into the chamber using a centrifugal ventilator and the warm air was cooled using cooling equipment and moved again into the chamber as in this case, cooling happens on a closed circuit.

Each experimental test, consisting in a cooling and heating cycle, was repeated three times. All box's walls were of the same type in each experimental test. This condition allows the use of average values that eliminate the influence of potential errors or sporadic anomalies. However, differences in ambient temperature were still detected. The stack of boxes was placed near the center of the chamber as shown in Figure 2. 


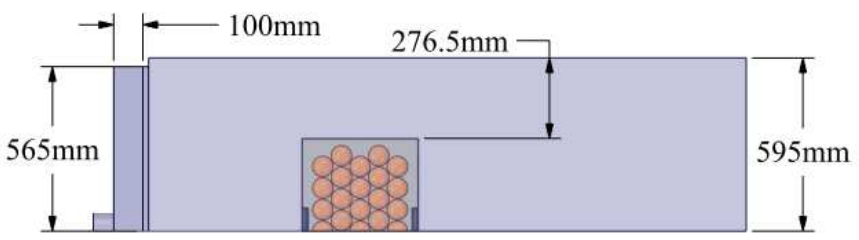

(a) Top view.

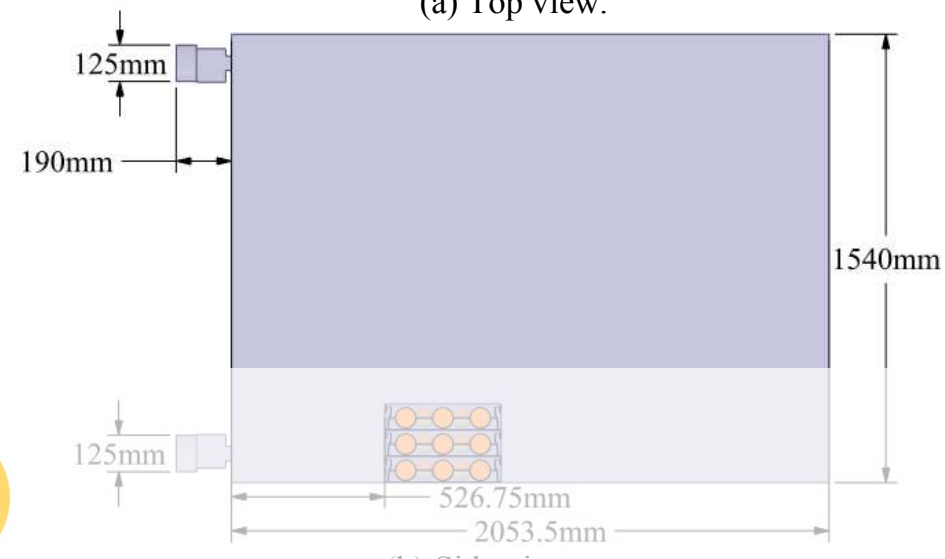

(b) Side view.

Figure 2: Placement of packaging boxes inside the cooling chamber.

3 NUMERICAL MODELS

3.1 Governing Equation

The mathematical $m$

equations of conserva

conservation of energy (Eq. 3)[35]:
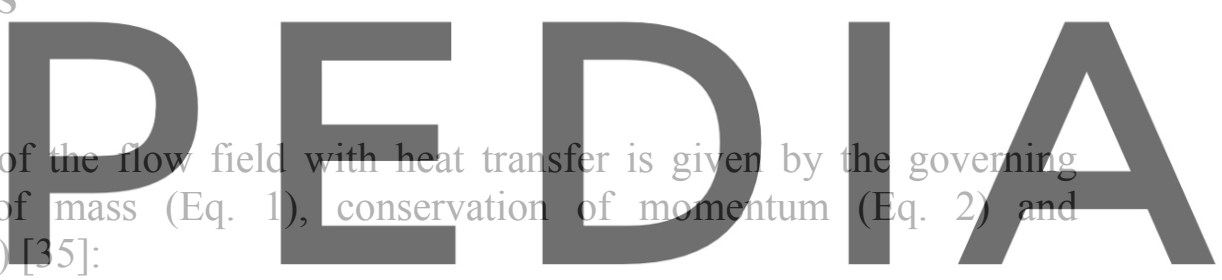

Register for free at https//www.scipedia.com(ta download the version without the waitermark

$$
\begin{gathered}
\frac{\partial \rho \mathrm{u}}{\partial \mathrm{t}}+\nabla \cdot(\rho \mathrm{uu})=-\nabla \mathrm{p}+\nabla \cdot \tau+\mathrm{F} \\
\frac{\partial}{\partial \mathrm{t}}\left[\rho\left(\mathrm{e}+\frac{1}{2} \mathrm{u}^{2}\right)\right]+\nabla \cdot\left[\rho \mathrm{u}\left(\mathrm{e}+\frac{1}{2} \mathrm{u}^{2}\right)\right]=\nabla \cdot(\mathrm{k} \nabla \mathrm{T})+\nabla \cdot(-\mathrm{pu}+\tau \cdot \mathrm{u})+\mathrm{u} \cdot \mathrm{F}+\mathrm{Q}
\end{gathered}
$$

Considering the results obtained by Defraeye et al. [32], the standard k- $\omega$ was chosen for turbulent flow simulations. It uses the transport equation for the turbulent kinetic energy (Eq. 4) and for its specific dissipation rate (Eq. 5). Air density is determined by the ideal gas law for incompressible flow (Eq. 6).

$$
\begin{gathered}
\frac{\partial}{\partial \mathrm{t}}(\rho \mathrm{k})+\frac{\partial}{\partial \mathrm{x}_{\mathrm{i}}}\left(\rho \mathrm{ku}_{\mathrm{i}}\right)=\frac{\partial}{\partial \mathrm{x}_{\mathrm{j}}}\left(\Gamma_{\mathrm{k}} \frac{\partial \mathrm{k}}{\partial \mathrm{x}_{\mathrm{j}}}\right)+\mathrm{G}_{\mathrm{k}}-\mathrm{Y}_{\mathrm{k}}+\mathrm{S}_{\mathrm{k}} \\
\frac{\partial}{\partial \mathrm{t}}(\rho \omega)+\frac{\partial}{\partial \mathrm{x}_{\mathrm{i}}}\left(\rho \omega \mathrm{u}_{\mathrm{i}}\right)=\frac{\partial}{\partial \mathrm{x}_{\mathrm{j}}}\left(\Gamma_{\omega} \frac{\partial \omega}{\partial \mathrm{x}_{\mathrm{j}}}\right)+\mathrm{G}_{\omega}-\mathrm{Y}_{\omega}+\mathrm{S}_{\omega}
\end{gathered}
$$




$$
\rho=\frac{\mathrm{p}_{\mathrm{op}}}{\frac{\mathrm{R}}{\mathrm{M}_{\mathrm{w}}} \mathrm{T}}
$$

\subsection{Material properties}

The simulations required to set the properties of several materials: Polypropylene for the boxes, polystyrene for some of the product simulators, agar-gel for the remainder of the product simulators, carton board for the alveoli and dry air for the fluid domain. All properties were considered constant during the simulation, except for air density, due to small variations in their value within the temperature range. Properties are shown in Table 1 [36-37].

Table 1: Thermal and physical properties of the materials used.

\begin{tabular}{|c|c|c|c|c|c|}
\hline Material & $\begin{array}{c}\text { Density } \\
{\left[\mathrm{kg} / \mathrm{m}^{3}\right]}\end{array}$ & $\begin{array}{c}\text { Thermal } \\
\text { conductivity } \\
{[\mathrm{W} / \mathrm{m} \cdot \mathrm{K}]}\end{array}$ & $\begin{array}{c}\text { Specific } \\
\text { heat } \\
{[\mathrm{J} / \mathrm{kg} \cdot \mathrm{K}]}\end{array}$ & $\begin{array}{c}\text { Viscosity } \\
{[\mathrm{kg} / \mathrm{m} \cdot \mathrm{s}]}\end{array}$ & $\begin{array}{c}\text { Molecular } \\
\text { weight } \\
{[\mathrm{kg} / \mathrm{kmol}]}\end{array}$ \\
\hline Polypropylene & 800 & 0.15 & 1200 & --- & --- \\
\hline Polystyrene & 16 & 0.04 & 1210 & --- & --- \\
\hline Agar-gel $(2 \% \mathrm{~m} / \mathrm{y})$ & 1000 & 0.55 & 4198 & --- & --- \\
\hline Carton board & 930 & 0.18 & 1340 & --- & 28.97 \\
\hline Air & Eq. 6 & 0.02 & 1007 & $1.72 \times 10^{-5}$ & \\
\hline
\end{tabular}

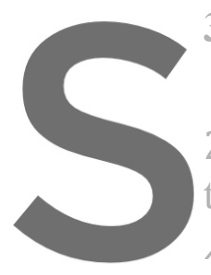

\subsection{Simulation setup}

Two unstructured $2.6 \times 10^{6}$ elements

temperature values, takcton $\lambda=1 \times 10^{-4}$ for resid
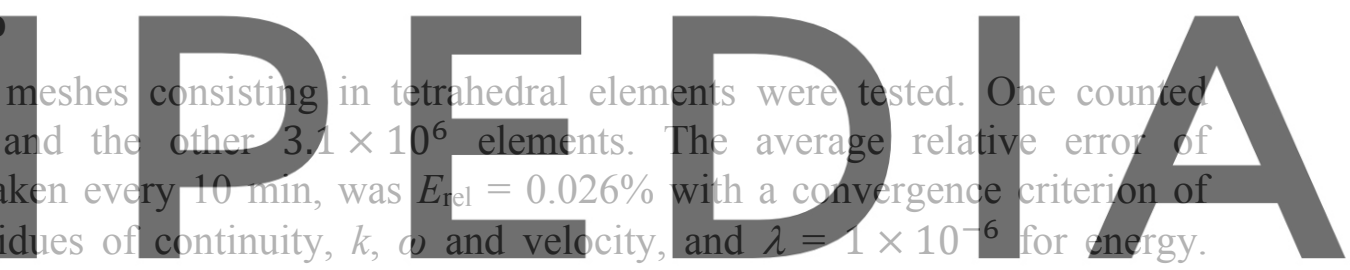

Therefore, the simulations were made using the coarser mesh (see Figure 3) but with the

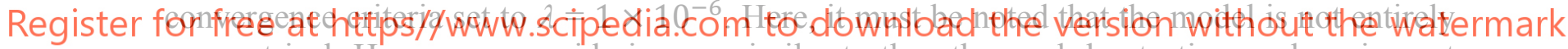
symmetrical. However, one side is very similar to the other and due to time and equipment restraints it is not possible to simulate the entire chamber. Due to this circumstances, the models for the boxes and the alveoli have an excess of $18.5 \mathrm{~mm}$ inside the chamber per comparison to what would be the correct middle plane of the box.

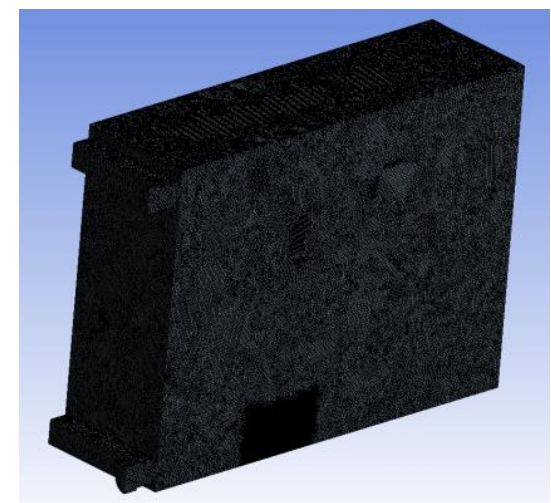

(a) Computational domain.

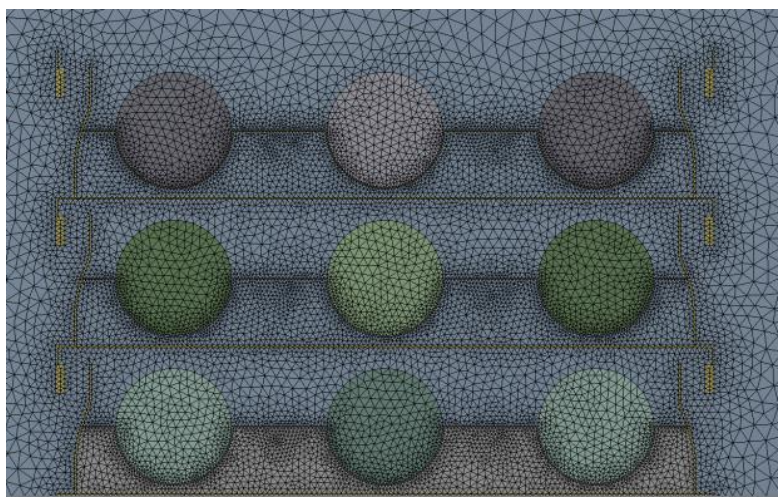

(b) Mesh detail.

Figure 3: Computational domain: (a) mesh of the full model, (b) close-up view on the symmetry plane. 
Two timesteps, $\Delta t_{1}=60$ and $\Delta t_{2}=120$ seconds, were also tested using the same criteria as described above. The difference in temperature measurements between them is $0.12 \%$. Therefore, the timestep of $\Delta t_{2}=120$ seconds was considered adequate for simulation.

All simulations were made using machines running the Windows 7 operating system, with 192 GB of RAM and using the commercial code Ansys Products 2019 R3.

The accuracy of CFD simulations depends on the turbulence model used in the ReynoldsAveraged Navier-Stokes equations. In this effect and in accordance to the findings of Defraeye et al. [29], the standard $k$ - $\omega$ was used for its compromise between computational requirements, computation time and accuracy. Due to symmetry, it becomes possible to slice the entire model in two halves and use only one for computation to reduce the time consumed by simulations. As for boundary conditions, the fluid domain represents half for the interior of the cooling chamber and consists in a velocity inlet with an entry velocity of $v_{\text {in }}=0.8 \mathrm{~m} / \mathrm{s}$ and a temperature of $T_{\text {in }}=2.5^{\circ} \mathrm{C}$, a pressure outlet with a pressure differential equal to ambient pressure at sea level. The outer wall of the fluid domain was defined as adiabatic to represent the thick insulation layer of the cooling chamber, and the middle wall was defined as a symmetry plane. For the solid domain, all walls in contact with the fluid domain were set as coupled except for the ones in the symmetry face, which too were defined as symmetry. All domains were set to an initial ambient temperature of $T_{\mathrm{amb}}=22^{\circ} \mathrm{C}$. The SIMPLEC method was used for the pressure linked equations. Second order formulation was used for all parameters at the exception of transient formulation which was left as first order. The transient analysis was perforned with $n=240$ timesteps, $\Delta t=120$ seconds each and $i=20$
iterations per timestep. Simulating tfing $=8$ hours of cooling took approximately $t_{\text {total }}=60$ to
70 hours per simulation, depending on the packaging box model.
4 RESULTS AND DISCUSSION

4.1 Numerical validation

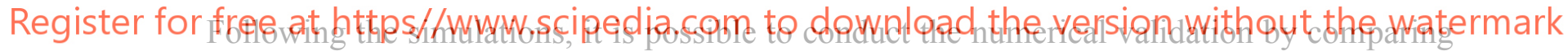
the temperature data at the core of the agar spheres for the simulation and the average data of the experimental tests conducted by [34].

\subsubsection{Model A}

In the bottom box it is possible to verify an extremely good adjustment between experimental and numerical data. The total temperature variation was of $\Delta T_{\text {num }}=11.6^{\circ} \mathrm{C}$ for the CFD simulation and $\Delta T_{\exp }=11.8^{\circ} \mathrm{C}$ for the average of the experimental tests. The temperature variation for the 8-hour simulation has a time averaged relative error of $E_{\text {rel }}=1.5 \%$.

In the middle box, for the central sphere, some deviation from the experimental results was determined as the total temperature was $\Delta T_{\exp }=13.8^{\circ} \mathrm{C}$ and $\Delta T_{\text {num }}=10.1^{\circ} \mathrm{C}$, for experimental tests and simulation, respectively. The time averaged relative error for this sphere is $E_{\text {rel }}=19.3 \%$. Conversely, the lateral sphere in the middle box showed a very good adjustment as the experimental temperature drop was $\Delta T_{\exp }=12.8^{\circ} \mathrm{C}$ versus the simulated one, $\Delta T_{\text {num }}=12.6^{\circ}$ and a time averaged relative error of $E_{\text {rel }}=3 \%$.

In the top box, lidless and freely exposed to cold air, some discrepancy between 
experimental and numerical data was determined. The total temperature decrease was $\Delta T_{\exp }=15.5^{\circ} \mathrm{C}$ for the experimental test but only $\Delta T_{\text {num }}=13.2^{\circ} \mathrm{C}$ for the simulation. As the temperatures during the simulation were consistently higher than the experimental ones, the time averaged relative error for this sphere is $E_{\text {rel }}=18.1 \%$. Table 2 summarizes the data for this model.

Table 2: Simulation summary of a stack of model A boxes.

\begin{tabular}{|c|c|c|c|}
\hline Sphere location & $\Delta T_{\exp }\left[{ }^{\circ} \mathrm{C}\right]$ & $\Delta T_{\text {num }}\left[{ }^{\circ} \mathrm{C}\right]$ & $E_{\text {rel }}[\%]$ \\
\hline Bottom & 11.8 & 11.6 & 1.5 \\
\hline Central middle & 13.8 & 10.1 & 19.3 \\
\hline Lateral middle & 12.8 & 12.6 & 3.0 \\
\hline Top & 15.5 & 13.2 & 18.1 \\
\hline
\end{tabular}

\subsubsection{Model B}

For the sphere in the bottom box, a worse adjustment with the experimental results is determined when comparing with models A and C. In the simulation, this sphere cooled significantly more that in the experimental test and thus has a time averaged relative error of $E_{\text {rel }}=13.2 \%$ and simulated temperature drop of $\Delta T_{\text {num }}=13.3^{\circ} \mathrm{C}$ versus an experimental temperature drop of $\Delta T_{\exp }=11.7^{\circ} \mathrm{C}$. However, the match between the real and simulated values is much better than model $\mathrm{A}$ and $\mathrm{C}$ for the central sphere while maintain approximately

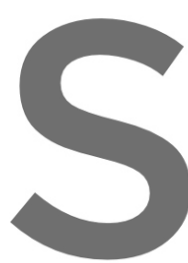
the same adjustment in $\Delta T_{\exp }=14.3^{\circ} \mathrm{C}$ versus relative error of $E_{\text {rel }}=$ achieved and is only error of $E_{\text {rel }}=3.5 \%$.
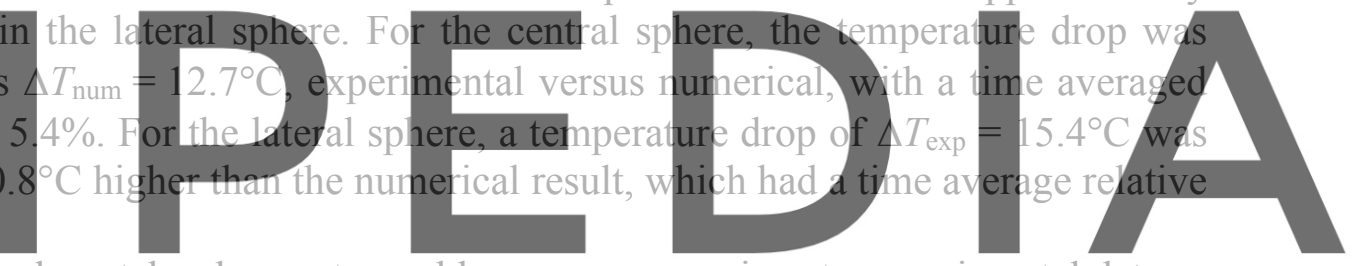

In the top box, the sphere takes longer to cooldown per comparison to experimental data as

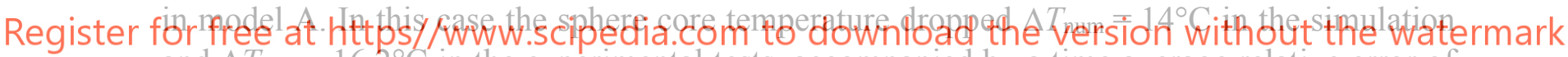
and $\Delta T_{\exp }=16.2^{\circ} \mathrm{C}$ in the experimental tests, accompanied by a time average relative error of $E_{\mathrm{rel}}=4.6 \%$. Table 3 summarizes the cooling data for this model.

Table 3: Simulation summary of a stack of model B boxes.

\begin{tabular}{|c|c|c|c|}
\hline Sphere location & $\Delta T_{\exp }\left[{ }^{\circ} \mathrm{C}\right]$ & $\Delta T_{\text {num }}\left[{ }^{\circ} \mathrm{C}\right]$ & $E_{\text {rel }}[\%]$ \\
\hline Bottom & 11.7 & 13.3 & 13.2 \\
\hline Central middle & 14.3 & 12.7 & 5.4 \\
\hline Lateral middle & 15.4 & 14.6 & 3.5 \\
\hline Top & 16.2 & 14.0 & 4.6 \\
\hline
\end{tabular}

\subsubsection{Model C}

Following the trend of model A, the sphere located in the bottom box of model $\mathrm{C}$ stack shows a very good adjustment to real data over time. The time averaged relative error is $E_{\text {rel }}=4 \%$ with a temperature drop of $\Delta T_{\exp }=11.8^{\circ} \mathrm{C}$ and $\Delta T_{\text {num }}=12.0^{\circ} \mathrm{C}$ for the experimental and numerical results, respectively.

In the middle box the behavior of the middle box in model $\mathrm{A}$ is observed. For the central 
sphere there is some discrepancy between experimental and numerical data but for the lateral sphere the adjustment is very satisfactory. For the central sphere, a time averaged error of $E_{\text {rel }}=16.6 \%$ was obtained with a temperature reduction of $\Delta T_{\exp }=13.8^{\circ} \mathrm{C}$ and $\Delta T_{\text {num }}=10.5^{\circ} \mathrm{C}$ concerning experimental and numerical data, respectively. The lateral sphere suffered a temperature drop of $\Delta T_{\exp }=12.8^{\circ} \mathrm{C}$ in both experimental tests and numerical simulations and it reached a time averaged relative error of $E_{\text {rel }}=3.7 \%$.

In the top box a similar behavior to model $\mathrm{A}$ is again detected, meaning a worse adjustment between numerical and experimental data. A temperature drop of $\Delta T_{\exp }=15.5^{\circ} \mathrm{C}$ was measured in the experimental tests but the temperature reduction for numerical simulation was only of $\Delta T_{\text {num }}=13.2^{\circ} \mathrm{C}$ and the time averaged relative error was $E_{\text {rel }}=17.1 \%$ as shown in Table 4.

Table 4: Simulation summary of a stack of model $\mathrm{C}$ boxes.

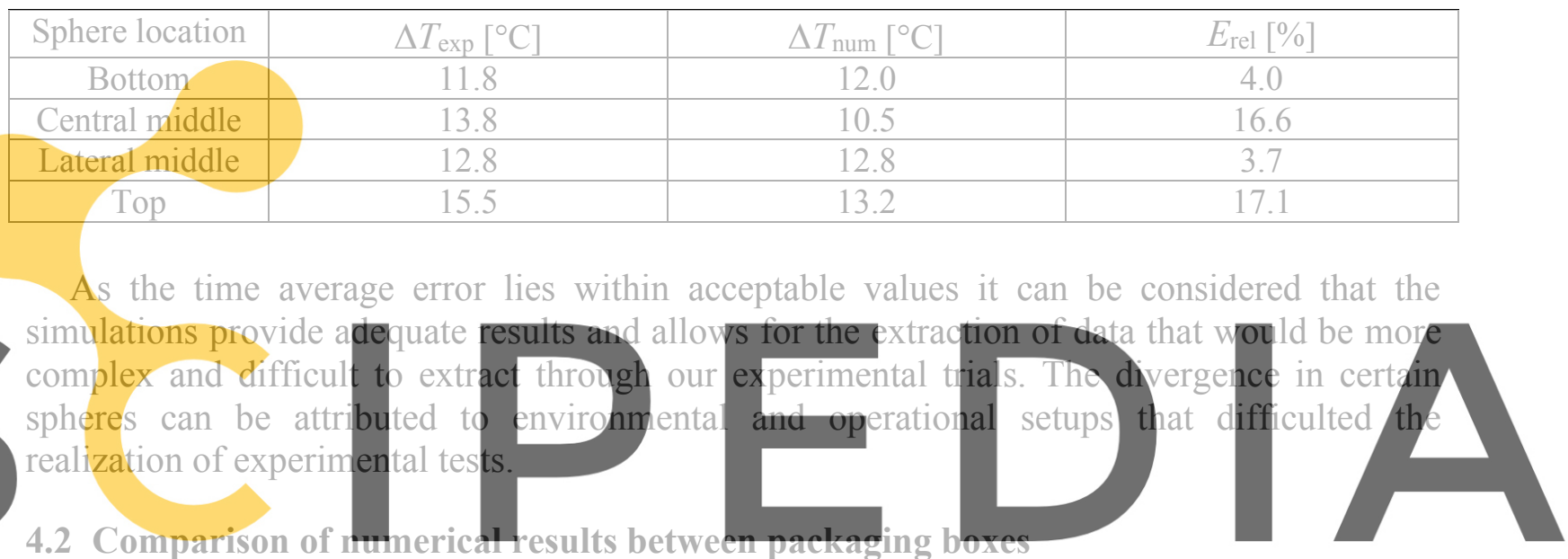

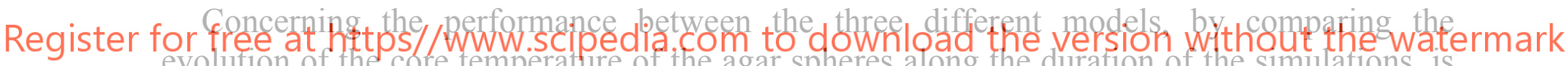 evolution of the core temperature of the agar spheres along the duration of the simulations, is} becomes very clear that the larger openings of model B are more effective at cooling, especially concerning the middle box. The prediction of the temperature evolution in the agar spheres is shown in Figure 3. Table 5 and Table 6 shows the predicted final temperature and half-cooling time. All data is expressed in dimensionless temperature and time to promote the results comparison between models.

Concerning the bottom box, model B is clearly superior while the models $\mathrm{A}$ and $\mathrm{C}$ show very similar characteristics. Model B clearly predicts lower temperatures and cooling time. The lateral sphere in the middle box benefits the most in terms of cooling due to the well distributed openings along the walls of all models. This allows for an even airflow along most of the box. Comparing the results with the central sphere in the middle box, it possible to observe that the spheres located along the airflow path in the zone of the handles of the boxes are less cooled than the rest. Still, model B is the clear best performer out of the three models.

The sphere located in the top box favors the model B per comparison with the others but not significantly. This is due to the exposure of this sphere to more circulating cold air as this box is uncovered. All this data is shown in Figure 4 and Tables 5 and 6. 


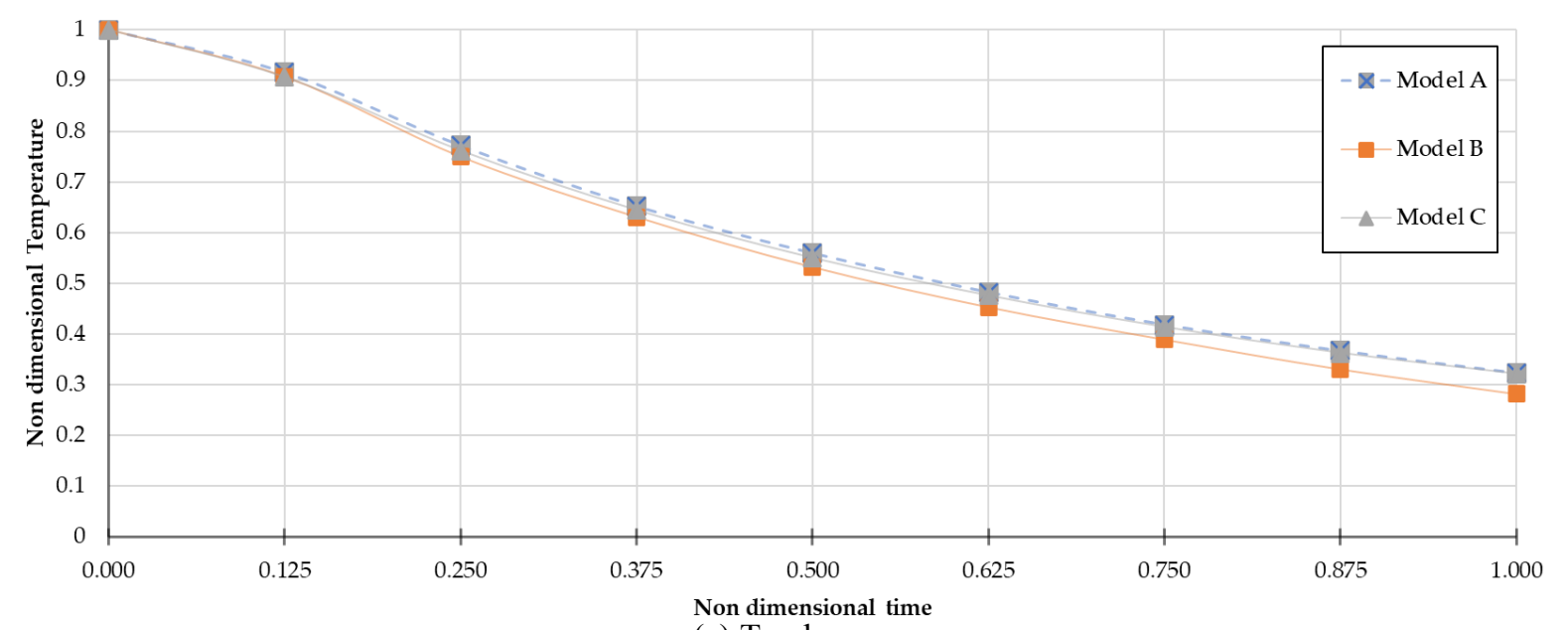

(a) Top box.
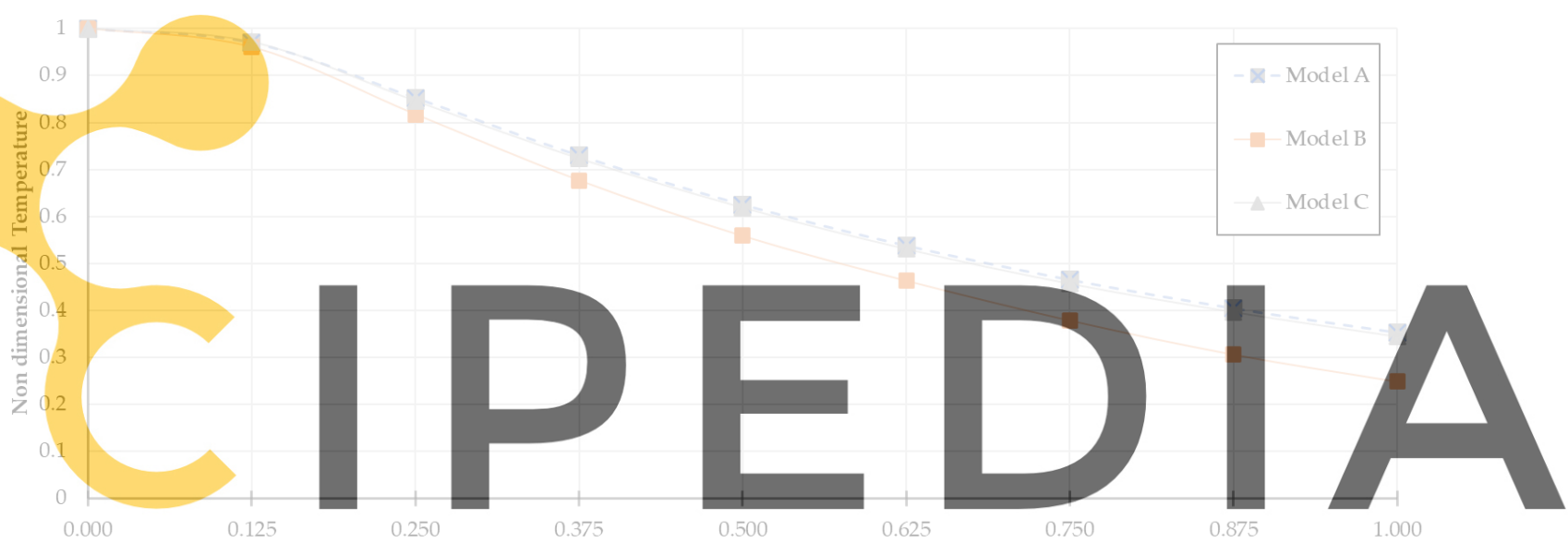

Register for free at https//www.scipedia.com toddowanload the version without the watermark

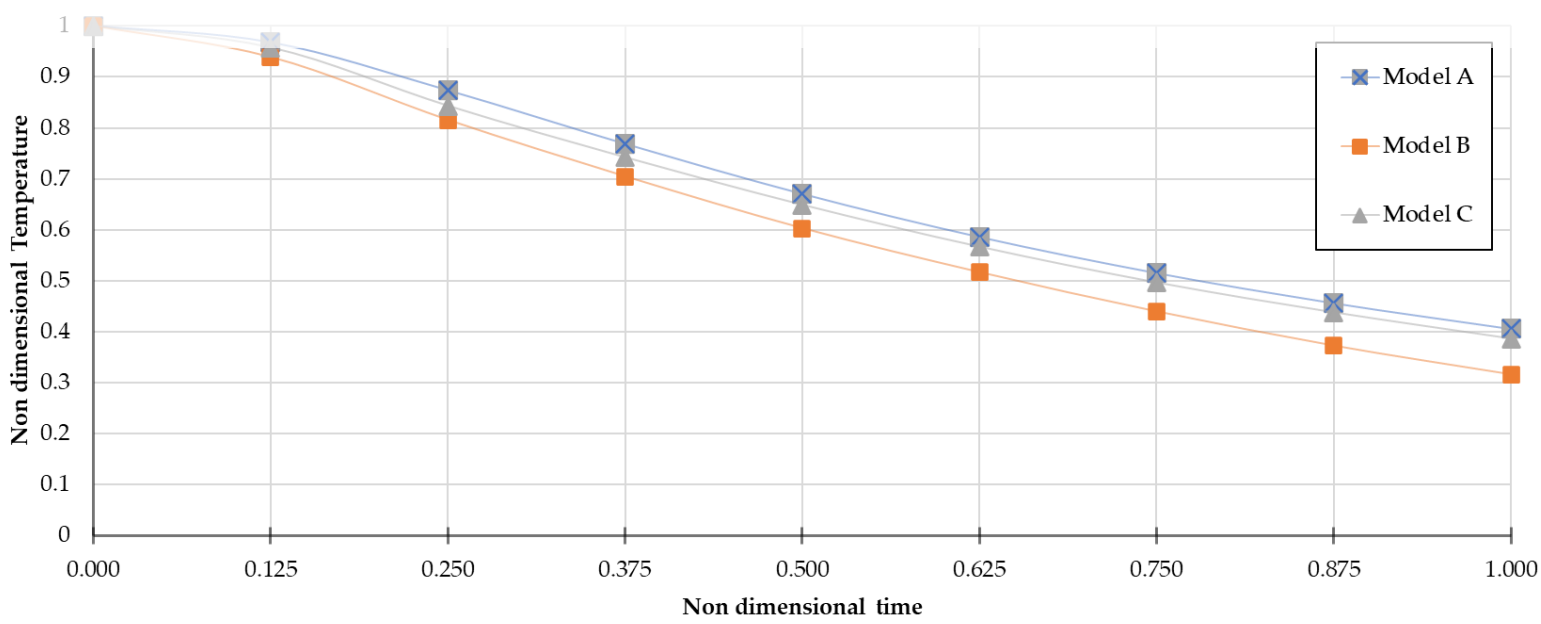

(c) Bottom box.

Figure 4: Time variation of non-dimensional temperature of agar spheres in the three boxes. 
Table 5: Non dimensional comparison of final temperature of agar sphere at different boxes.

\begin{tabular}{|l|c|c|c|}
\hline Box location & Model A & Model B & Model C \\
\hline Top & 0.32 & 0.28 & 0.32 \\
\hline Middle & 0.35 & 0.25 & 0.35 \\
\hline Bottom & 0.41 & 0.32 & 0.39 \\
\hline
\end{tabular}

Table 6: Non dimensional comparison of half cooling times (HTC) of agar sphere at different boxes.

\begin{tabular}{|l|c|c|c|}
\hline Box location & Model A & Model B & Model C \\
\hline Top & 0.60 & 0.56 & 0.59 \\
\hline Middle & 0.69 & 0.57 & 0.68 \\
\hline Bottom & 0.79 & 0.65 & 0.75 \\
\hline
\end{tabular}

Following these results, the distribution of temperature in the packaging boxes on the symmetry plane is shown in Figure 5 and the distribution of air temperature and velocity in the middle box, immediately above the spheres is shown in Figure 6.

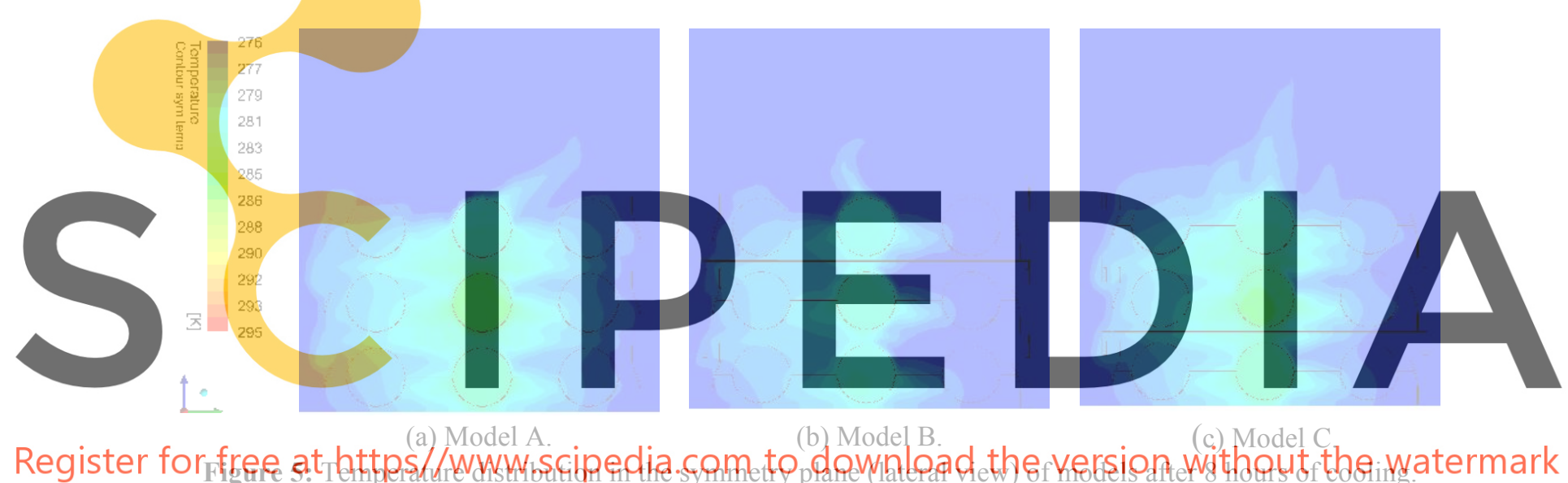

It is clear that model A does not allow for higher air velocity inside the box, which proves to be detrimental for the temperatures of the agar spheres.

For model B, it can clearly be predicted the influence of the larger openings as they allow for an increased flowrate through the spherical simulators thus justifying for lower temperatures and cooling times. It is also clearly visible the restriction in the airflow for the central sphere in the middle box due to the handle position.

Model C displays a very similar behavior to box A. It is another clear demonstration that even though the vents are uniformly distributed throughout the box, their small size restricts airflow through the spheres.

In general terms, all boxes deal with very low airflow velocity due to the restrictions imposed by the boxes in the chamber. Thus, in this scenario, a very significant bypass flow exists in the side of the boxes. Still, it is possible to predict that larger openings have a very positive influence in the airflow crossing the spheres. Whereas in model B it is possible to predict the negative effect of the handle position on the central middle sphere, that effect is not noticeable in the remaining models, due to the lower air velocity, implying higher 
temperatures at the end of the cooling operation with a broader high temperature zone.

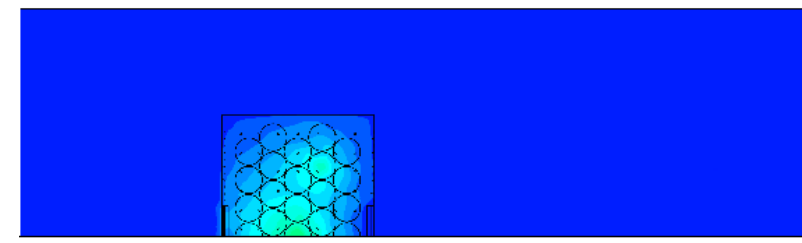

(a1) Temperature contour.

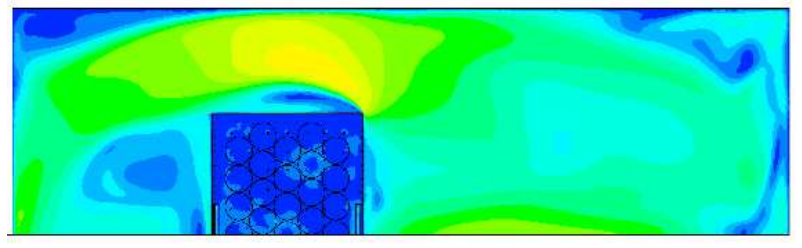

(a2) Velocity contour.

(a) Model A.

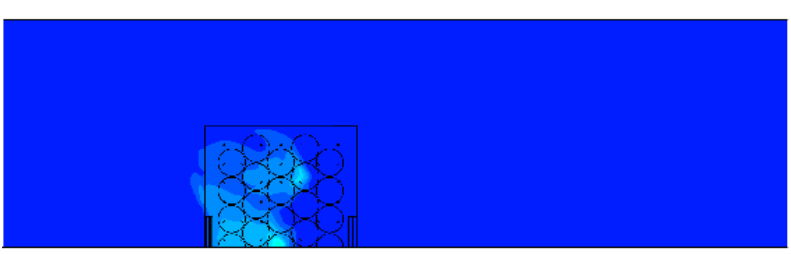

(b1) Temperature contour.

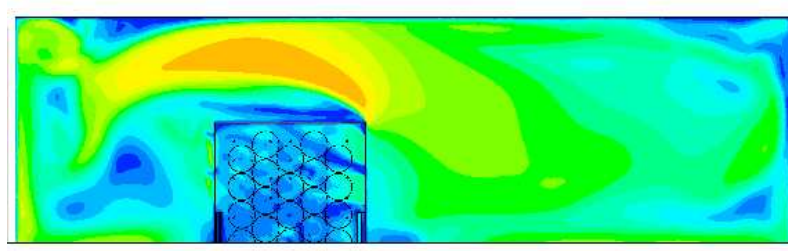

(b2) Velocity contour.

(b) Model B.
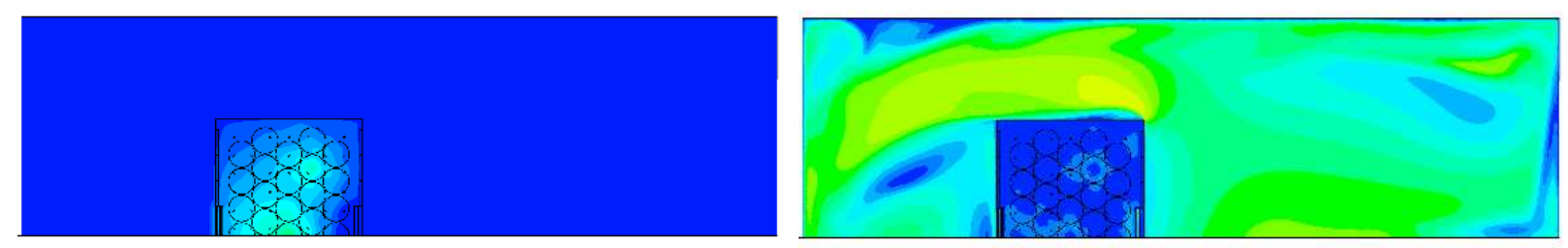

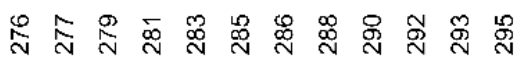

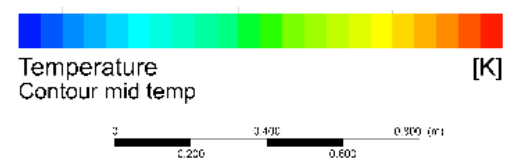

(c1) Temperature contour.

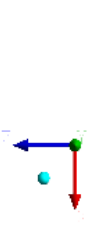

(c) Model C.
过

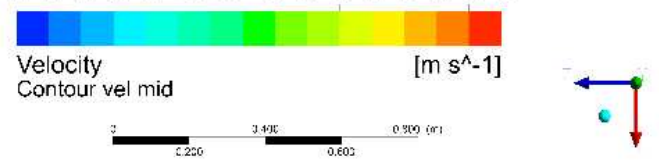

(c2) Velocity contour.

Figure 6: Temperature and velocity distribution in the middle plane (top view) of models after 8 hours of cooling.

\section{CONCLUSIONS}

As temperature is extremely important for the appropriate conservation of fresh postharvest fruits, it is necessary to develop new methods that allow for this condition. As packaging is one of the easiest to change, it is very worthwhile to invest in the design of highperformance packaging.

This study clearly demonstrates that larger and well distributed openings allow for more cooling efficiency and uniformity and that small openings perform only marginally better than larger opening distributed through anterior and posterior walls as they act as a blockade for the streams of cool air that is necessary to decrease fruit temperature.

In the future, it is intended to follow these results and analyze the cooling performance of 
these models but filled entirely of agar-gel simulators, to also analyze the heating performance of the models and to test the effects of phase change materials in fruit conservation.

\section{ACKNOWLEDGMENTS}

This study is within the activities of project "Pack2Life - High performance packaging", project IDT in consortium n. ${ }^{\circ} 33792$, call n. ${ }^{\circ}$ 03/SI/2017, Ref. POCI-01-0247-FEDER033792, promoted by COMPETE 2020 and co-funded by FEDER within Portugal 2020.

The authors thank the opportunity and financial support to carry on this project to Fundação para a Ciência e Tecnologia (FCT) and R\&D Unit "Centre for Mechanical and Aerospace Science and Technologies" (C-MAST), under project UIDB/00151/2020.

\section{REFERENCES}

[1] J. L. O'Sullivan, M. J. Ferrua, R. Love, P. Verboven, B. Nicolaï, and A. East, "Forced-air cooling of polylined horticultural produce: Optimal cooling conditions and package design," Postharvest Biol. Technol., 126, pp. 67-75, 2017, doi: 10.1016/j.postharvbio.2016.11.019.

[2] W. Gruyters et al., "Reusable boxes for a beneficial apple cold chain: A precooling analysis," Int. J. Refrig., 106, pp. 338-349, 2019, doi: 10.1016/j.ijrefrig.2019.07.003.

[3] L. Lu, X. Chen, and J. Wang, "Modelling and Thermal Analysis of Tray-layered Fruits inside Ventilated Packages during Forced-air Precooling," Packag. Technol. Sci., 29, pp. 105-119, 2016, doi: 10.1002/pts.

[4] H. Nalbandi, S. Seiiedlou, H. R. Ghasemzadeh, and F. Rangbar, "Innovative Parallel Airflow System for forced-air cooling of strawberries," Food Bioprod. Process., 100, pp. 440-449, 2016, doi: 10.1016/j.fbp.2016.09.002.

[5] T. Brosnan and D. W. Sun, "Precooling techniques and applications for horticultural products - a review," Int. J. Refrig., 24(2), pp. 154-170, 2001, doi: 10.1016/S01407007(00)00017-7.

[6] A. Ambaw, M. Mukama, and U. L. Opara, "Analysis of the effects of package design on the rate and uniformity of cooling of stacked pomegranates: Numerical and experimental studies," Comput. Electron. Agric., 136, pp. 13-24, 2017, doi: 10.1016/j.compag.2017.02.015.

[7] T. Defraeye et al., "Towards integrated performance evaluation of future packaging for fresh produce in the cold chain," Trends Food Sci. Technol., 44(2), pp. 201-225, 2015, doi: 10.1016/j.tifs.2015.04.008.

[8] A. Hafez et al., "Simplified Heat and Mass Transfer Modeling for Anna Apples Cold Storage," Int. J. Food Eng. Technol., 3(2), pp. 15-27, 2017, doi: 10.11648/j.ijfet.20170302.12.

[9] S. Akdemir and T. Bartzanas, "Numerical modelling and experimental validation of a cold store ambient factors," Tarim Bilim. Derg., 21(4), pp. 606-619, 2015, doi: 10.1501/tarimbil_0000001361.

[10] W. Gruyters et al., "Modelling Cooling of Packaged Fruit Using 3D Shape Models," Food Bioprocess Technol., 11(11), pp. 2008-2020, 2018, doi: 10.1007/s11947-018-2163-9.

[11] R. Jedermann and W. Lang, "Computational fluid dynamics modelling of deviating airflow and cooling conditions in banana containers," Acta Hortic., 1154, pp. 193-200, 2017, doi: 
10.17660/ActaHortic.2017.1154.25.

[12] T. Defraeye et al., "Artificial fruit for monitoring the thermal history of horticultural produce in the cold chain," J. Food Eng., 215, pp. 51-60, 2017, doi: 10.1016/j.jfoodeng.2017.07.012.

[13] P. B. Pathare, U. L. Opara, C. Vigneault, M. A. Delele, and F. A. J. Al-Said, "Design of Packaging Vents for Cooling Fresh Horticultural Produce," Food Bioprocess Technol., 5(6), pp. 2031-2045, 2012, doi: 10.1007/s11947-012-0883-9.

[14] W. Wu and T. Defraeye, "Identifying heterogeneities in cooling and quality evolution for a pallet of packed fresh fruit by using virtual cold chains," Appl. Therm. Eng., 133, pp. 407417, 2018, doi: 10.1016/j.applthermaleng.2017.11.049.

[15] G. Alvarez and D. Flick, "Analysis of heterogeneous cooling of agricultural products inside bins. Part I: Aerodynamic study," J. Food Eng., 39(3), pp. 227-237, 1999, doi: 10.1016/S0260-8774(98)00166-6.

[16] G. Alvarez and D. Flick, "Analysis of heterogeneous cooling of agricultural products inside bins. Part II: Thermal study,” J. Food Eng., 39(3), pp. 239-245, 1999, doi: 10.1016/S0260-8774(98)00166-6.

[17] J. Dehghannya, M. Ngadi, and C. Vigneault, "Mathematical modeling of airflow and heat transfer during forced convection cooling of produce considering various package vent areas," Food Control, 22(8), pp. 1393-1399, 2011, doi: 10.1016/j.foodcont.2011.02.019.

[18] M. A. Delele, M. E. K. Ngcobo, S. T. Getahun, L. Chen, J. Mellmann, and U. L. Opara, "Studying airflow and heat transfer characteristics of a horticultural produce packaging system using a 3-D CFD model. Part I: Model development and validation," Postharvest Biol. Technol., 86, pp. 536-545, 2013, doi: 10.1016/j.postharvbio.2013.08.014.

[19] M. A. Delele, M. E. K. Ngcobo, S. T. Getahun, L. Chen, J. Mellmann, and U. L. Opara, "Studying airflow and heat transfer characteristics of a horticultural produce packaging system using a 3-D CFD model. Part II: Effect of package design," Postharvest Biol. Technol., 86, pp. 546-555, 2013, doi: 10.1016/j.postharvbio.2013.08.015.

[20] L. R. de Castro, C. Vigneault, and L. a. B. Cortez, "Container opening design for horticultural produce cooling efficiency," Food, Agric. Environ., 2(1), pp. 135-140, 2004.

[21] L. R. De Castro, C. Vigneault, and L. A. B. Cortez, "Effect of container opening area on air distribution during precooling of horticultural produce," Trans. Am. Soc. Agric. Eng., 47(6), pp. 2033-2038, 2004.

[22] L. R. De Castro, C. Vigneault, and L. A. B. Cortez, "Cooling performance of horticultural produce in containers with peripheral openings," Postharvest Biol. Technol., 38(3), pp. 254-261, 2005, doi: 10.1016/j.postharvbio.2005.07.004.

[23] T. M. Berry, T. Defraeye, B. M. Nicolaï, and U. L. Opara, "Multiparameter Analysis of Cooling Efficiency of Ventilated Fruit Cartons using CFD: Impact of Vent Hole Design and Internal Packaging," Food Bioprocess Technol., 9(9), pp. 1481-1493, 2016, doi: 10.1007/s11947-016-1733-y.

[24] T. M. Berry, T. S. Fadiji, T. Defraeye, and U. L. Opara, "The role of horticultural carton vent hole design on cooling efficiency and compression strength: A multi-parameter approach," Postharvest Biol. Technol., 124, pp. 62-74, 2017, doi: 10.1016/j.postharvbio.2016.10.005.

[25] B. A. Anderson, A. Sarkar, J. F. Thompson, and R. P. Singh, "Commercial-scale forced-air cooling of packaged strawberries," Trans. Am. Soc. Agric. Eng., 47(1), pp. 183-190, 2004, doi: $10.13031 / 2013.15846$.

[26] W. Wu, P. Häller, P. Cronjé, and T. Defraeye, "Full-scale experiments in forced-air 
precoolers for citrus fruit: Impact of packaging design and fruit size on cooling rate and heterogeneity," Biosyst. Eng., 169, pp. 115-125, 2018, doi: 10.1016/j.biosystemseng.2018.02.003.

[27] T. Defraeye et al., "Forced-convective cooling of citrus fruit: Package design," J. Food Eng., 118(1), pp. 8-18, 2013, doi: 10.1016/j.jfoodeng.2013.03.026.

[28] J. W. Han, C. J. Zhao, J. P. Qian, L. Ruiz-Garcia, and X. Zhang, "Numerical modeling of forced-air cooling of palletized apple: Integral evaluation of cooling efficiency," Int. $J$. Refrig., 89, pp. 131-141, 2018, doi: 10.1016/j.ijrefrig.2018.02.012.

[29] T. Defraeye, P. Verboven, and B. Nicolai, "CFD modelling of flow and scalar exchange of spherical food products: Turbulence and boundary-layer modelling," J. Food Eng., 114(4), pp. 495-504, 2013, doi: 10.1016/j.jfoodeng.2012.09.003.

[30] A. Ilangovan, P.D. Gaspar, P.D. Silva, "Airflow and thermal behavior within peachs packaging box using Computational Fluid Dynamics - A preliminary study", KnE Engineering, 5(6), pp. 222-231, 2020.

[31] A. Ilangovan, P.D. Gaspar, P.D. Silva, P. Parente, "A parametric study and performance evaluation of the different vent hole configuration for fruit packaging using Computational Fluid Dynamics," 6th IIR International Conference on Sustainability and the Cold Chain (ICCC 2020). Nantes, France, August, 26-28, 2020, 346-353.

[32] A. Ilangovan, P.D. Gaspar, P.D. Silva, A.R. Gonçalves, A.M. Sampaio, A.J. Pontes, N. Alves, "CFD parametric study of. thermal performance of different fruit packaging box designs," AIP Conference Proceedings, 15th International Symposium on Numerical Analysis of Fluid Flows, Heat and Mass Transfer - Numerical Fluids 2020 (ICNAAM 2020), Rhodes, Greece, 17-23 September, 2020.

[33] S.K. Madham, F. Leitão, P.D. Silva, P.D. Gaspar, D. Duarte, "Experimental tests of the thermal behaviour of new sustainable bio-packaging food boxes," Procedia Environmental Science, Engineering and Management 8(1), pp. 215-223, 2021.

[34] F. Leitão, P.D. Silva, P.D. Gaspar, L.C. Pires, A.R. Gonçalves, D. Duarte, "Experimental study of thermal performance of different fruit packaging box designs," Energies 2021.

[35] S. Patankar and D. Spalding, "A calculation procedure for heat, mass and momentum transfer in three-dimensional parabolic flows," International Journal of Heat and Mass Transfer, 15(10), pp.1787-1806, 1972.

[36] T.L. Bergman, A.S. Lavine, F.P. Incropera, D.P. DeWitt, "Fundamentals of Heat and Mass Transfer," 8th ed.; John Wiley and Sons Inc, 2017.

[37] M. Zhang, Z. Che, J. Chen, H. Zhao, L. Yang, Z. Zhong, J. Lu, "Experimental determination of thermal conductivity of water-agar gel at different concentrations and temperatures," Journal of Chemical \& Engineering Data, 56, pp. 859-864, 2011. 